\title{
Théologiques
}

Théologiques

\section{Le concept de martyre en islam}

\section{Ali G. Dizboni}

Volume 13, numéro 2, automne 2005

Violence et souffrance rédemptrices

URI : https://id.erudit.org/iderudit/013605ar

DOI : https://doi.org/10.7202/013605ar

Aller au sommaire du numéro

\section{Éditeur(s)}

Faculté de théologie et de sciences des religions, Université de Montréal

ISSN

1188-7109 (imprimé)

1492-1413 (numérique)

Découvrir la revue

\section{Citer cet article}

Dizboni, A. G. (2005). Le concept de martyre en islam. Théologiques, 13(2), 69-81. https://doi.org/10.7202/013605ar

\section{Résumé de l'article}

Cet article analyse le concept de martyre en islam. En premier lieu, nous présentons brièvement le concept de djihad selon le Coran, les hadiths (les paroles de Mahomet) et les propos des compagnons de ce dernier. En deuxième lieu, nous explorons les caractéristiques du concept de martyre et son lien avec le djihad dans les textes sacrés. En troisième et dernier lieu, nous examinons plus spécifiquement certains aspects rituel, politique et idéologique du martyre dans l'islam et l'islamisme chiites contemporains.
Tous droits réservés $\odot$ Faculté de théologie et de sciences des religions, Université de Montréal, 2006
Ce document est protégé par la loi sur le droit d'auteur. L'utilisation des services d’Érudit (y compris la reproduction) est assujettie à sa politique d'utilisation que vous pouvez consulter en ligne.

https://apropos.erudit.org/fr/usagers/politique-dutilisation/ 


\section{Le concept de martyre en islam}

Ali G. Dizboni (Ph. D.)

Collège militaire du Canada

Kingston

La Révolution islamique de Khomeiny en 1979, la prolifération des mouvements islamistes dans un contexte de conflits régionaux, la kamikisation des opérations terroristes, l'antiaméricanisme croissant dans le monde musulman et les attentats terroristes du 11 septembre 2001 constituent quelques faits marquants des turbulences dans l'ordre international depuis les années 1980. Portés par une idéalisation de leur sacrifice, les «fous d'Allah» sont devenus l'incarnation de «l'axe du Mal». Leur action paraît insensée aux yeux de bien des Occidentaux qui y voient, entre autres choses, une instrumentalisation de la notion musulmane du martyre.

Qu'en est-il au juste du martyre dans l'islam ? Notre réponse veut aller au-delà des clichés et des lieux communs. À cet effet, notre travail s'articulera autour de plusieurs éléments. Le djihad et le martyre sont indissociables et constituent les deux côtés de la même médaille. Nous avons déjà abordé la question du djihad dans une étude récente (Dizboni 2003) que nous résumerons d'abord pour nous concentrer ensuite sur le statut du martyr et sur la signification du sacrifice physique et de la souffrance en islam, en particulier dans le chiisme. Cette analyse des spécificités de la notion de martyre chiite nous permettra de mieux comprendre le contexte contemporain de cette tradition.

\section{La période préislamique et la guerre}

Le Coran qualifie la période préislamique de jabiliyya (ignorance) vu la prédominance de l'idolâtrie et du polythéisme. La guerre intertribale est le modus operandi de cette société permettant ainsi au guerrier d'atteindre le sommet de la noblesse personnelle et de la vertu morale. Cette culture de guerre est due surtout à la rigueur climatique et à l'anarchie politique, donnant lieu à d'interminables guerres fratricides (Lammens 1914, 138). La 
tribu devient de ce fait le cadre primaire de l'identité et la défense de l'honneur tribal, la première cause de conflits. À ce sujet, Ibn Khaldoun, l'historien musulman du XII ${ }^{\mathrm{e}}$ siècle, a formulé la fameuse notion de asabyya, (esprit de corps), pour décrire ce chauvinisme tribal.

Les poèmes de la période de jahiliyya, les seules sources qui nous sont parvenues de l'époque préislamique, prêchent l'honneur tribal et la glorification de la guerre. Cette glorification atteint l'exaltation extrême pour dépasser la seule défense absolue de ses proches et devenir une fin en soi (Hitti 1968, 25).

\section{La période islamique et le discours de la guerre}

Avec l'arrivée de l'islam, certes, les règles, les objectifs et les formes de guerres ont changé; mais il n'y a pas de rupture totale avec la tradition guerrière préislamique au niveau de la valorisation de la guerre. L'établissement de la cité-État de Médine par Mahomet en 613 a réuni les tribus d'Arabie, mettant ainsi fin à l'anarchie politique. Le djihad pour la cause de l'expansion islamique prend le dessus sur le chauvinisme militariste

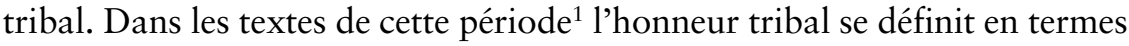
religieux et le lien de sang cède la place à la conviction religieuse. Cet esprit de guerre sainte a alimenté au moins 115 expéditions militaires et confrontations de toutes sortes - des manœuvres militaires jusqu'à de véritables guerres, en passant par des escarmouches (Ibn Hicham 1967, vol. 1). Mahomet lui-même en dirigea 27.

Le Coran, ainsi que les recueils classiques de hadith, les actes et les paroles de Mahomet recueillis en textes, sont catégoriques sur la vertu du djihad et sur la valeur du sacrifice physique (le martyre). La guerre est nécessaire au plan divin d'établissement de l'ordre sur terre et les musulmans ont l'obligation de s'y préparer. Cette sanctification de la guerre, de la mort sur le champ de bataille et de l'épreuve des souffrances, est soulignée à maintes reprises. L'exemple le plus frappant est le fait que le djihad devient un devoir de la foi et s'inscrit dans la loi islamique ${ }^{2}$ (charia), comme il est écrit dans le Coran: "Le combat vous a été prescrit mais vous avez de

1. Pour des citations, se référer à Dizboni 2003.

2. L'importance de l'obligation de la guerre ainsi que des récompenses promises au mujahid (combattant du djihad) est reprise par les juristes dès le II siècle de l'hégire (voir Sarakhsi 1989, 6-28). 
l'aversion pour lui, il se peut que vous haïssiez une chose qui est bonne pour vous...» (Coran 2,216).

La participation à la guerre devient ainsi une épreuve divine pour distinguer les vrais croyants des non-croyants (Coran 3,186; 47,31; 49,15). La preuve en est que l'échelle des valeurs coraniques privilégie l'obéissance au commandement du diihad (Coran 4,95). En lisant les hadiths, on se rend bien compte que le djihad suit immédiatement l'acte de témoignage de foi en Dieu (Nabhani 1984, 20). Le croyant qui combat dans la voie d'Allah, risquant sa vie et ses avoirs, est l'individu le plus sublime (Bukhari 1974, 24 ; Nabhani 1984, 22). Pour Mahomet, le djihad est une partie fondamentale de sa mission. Il dit dans un hadith très célèbre: "J'ai donné l'ordre de combattre ces gens [les infidèles] jusqu'à ce qu'ils témoignent qu'il n'y pas de dieux qu'Allah. S'ils le font leur sang et leur propriété sont sauvés» (Bukhari 1974, 265 ; Nabhani 1984, 20).

\section{Le martyre}

Sur le plan étymologique, l'expression souvent utilisée dans les hadiths et dans la charia pour désigner "la mort dans le chemin d'Allah» est chahada (martyre) et chahid (martyr). Dans le Coran, par contre, ces deux mots, utilisés sous différentes formes et à cinquante-six reprises, désignent plutôt le témoignage, et non la mort sacrée. Comme le dit Khosrokhavar (2003, 21), dans le Coran, chaque fois qu'il est question de mourir pour Allah, on emploie des expressions comme "périr sur le chemin de Dieu » $(2,154)$, «tomber sur le chemin de Dieu» $(3,157)$, «être tué sur le chemin de Dieu $(3,169)$, «combattre sur le chemin de Dieu» $(4,74)$, « faire exode sur le chemin de Dieu et y être tué ou mort» $(22,58)$.

La notion de martyre prend en islam une double acception. Le premier sens, plus général, vient de l'intention du croyant et de la nature de ses actes sans que la mort survienne obligatoirement sur un champ de bataille. Ainsi, par exemple, le décès naturel d'un pèlerin est qualifié de chahada. Le deuxième sens, plus spécifique, celui qui nous intéresse ici, réfère à la mort du croyant dans une guerre pour la cause d'Allah (djihad). Dans les hadiths et dans la charia, le mot chahid renvoie souvent à cette signification spécifique. Il y a certainement un consensus parmi les différentes écoles islamiques sur l'importance privilégiée accordée au martyr par le Coran, le hadith et les autres sources auxiliaires classiques (les interprétations du Coran, les histoires des guerres d'islam, les biographies des compagnons des prophètes, etc.). Le verset suivant renvoie à ce lien spécifique entre le 
djihad et le martyre: "Car combattre sur le chemin de Dieu, c'est obtenir ou mort ou victoire: dans les deux cas, nous lui vaudrons salaire magnifique.» (Coran 4,74) Cet autre verset approfondit ce lien en mettant l'accent sur le sacrifice physique et sur l'épreuve de souffrance:

Certes, Allah a acheté des croyants, leurs personnes et leurs biens en échange du paradis. Ils combattent dans le sentier d'Allah: ils tuent, et ils se font tuer. C'est une promesse authentique qu'il a prise sur Lui même dans la Thora, l'Évangile et le Coran. Et qui est plus fidèle qu'Allah à son engagement? Réjouissez-vous donc de l'échange que vous avez fait: et c'est là le très grand succès. $(9,111$, cité dans Khosrokhavar 2003, 22)

Comme nous pouvons aussi le constater, ce verset nous permet de dégager plus clairement le lien entre le martyre et le djihad, à savoir «tuer ou se faire tuer dans la voie d'Allah».

Parce que le Coran lui accorde une place singulière, le martyr atteint le summum de la perfection morale que peut incarner un homme. Il n'est précédé que par la place qu'occupe le prophète lui-même. Le Coran récompense cette perfection morale par la vie éternelle: "Ne dites pas de ceux qui sont tués dans la voie d'Allah: "ce sont des morts". Mais (dites): "ce sont des vivants”. Mais vous ne le percevez pas.» (Coran 2,154) Dans le fameux verset 169 de la sourate 3, nous pouvons lire: «Ne comptez pas ceux qui ont été tués dans la voie d'Allah pour des morts. Mais ils sont des vivants auprès de leur Seigneur, pourvus de tout ». De plus, comme il est écrit dans le Coran, le sacrifice physique est une preuve de l'authenticité de la foi du martyr en Dieu: "Vous les Juifs, si vous êtes les amis distingués d'Allah, souhaitez la mort, si vous dites la vérité. » $(62,6)$ Dans le livre sacré, le sacrifice physique est intimement lié à la conception de la patience (sabr) face à la souffrance dans la voie de Dieu; il est ainsi dit: «Pour que nous vous examinions afin d'identifier les mudjahedeen [combattants] et les patients» (47,31). Dans les hadiths, il n'y a aucune ambiguïté ou équivoque puisqu'on accorde au martyr le statut de sauveur qui peut demander le pardon pour ses proches au jour de la résurrection (Abu Dawud 1980, 15-16).

L'exaltation dans le texte coranique du martyr et sa valorisation prophétique ont aussi eu un effet très important dans la mobilisation militaire et dans la motivation psychologique des tribus arabes converties. Selon Klausner, "l'acte exemplaire du martyr renforce le courage des gens les aidant à supporter les tumultes quotidiens et dirige leur colère contre l'adversaire meurtrier et cruel» $(1987,230)$. La force psychologique que 
produit la doctrine du martyre est illustrée par les propos recueillis par l'historien Ibn Hicham de combattants musulmans du début de l'islam. Un premier combattant décrit la punition qui sera infligée aux infidèles: «Ils alimentent le feu de l'Enfer / Chaque infidèle doit y demeurer / le feu les consommera / sa chaleur s'excitera par les morceaux de fer et de pierre » (1967, 344). Un deuxième combattant décrit ses compagnons d'armes morts dans la guerre contre les polythéistes: "Ces morts sont dans le Sublime Paradis / honorés à son entrée et à sa sortie» (409). Un troisième mujahid illustre bien comment les principes du texte sacré se traduisent en attitudes et en comportements: «Si vous coupez mes jambes, je reste un musulman / j'espère, en échange, gagner une vie proche d'Allah - avec les houris [les belles anges du paradis] / habillées comme les plus belles statues avec le paradis suprême consacré à ceux qui y parviennent» (349).

À cette force psychologique s'ajoute la conviction que les soldats d'Allah sont invincibles. Cet endoctrinement vise à renforcer la détermination et à écarter tout traumatisme qu'une défaite pourrait infliger dans l'esprit des croyants. Les combattants musulmans pourront ainsi atteindre « une des deux meilleures [choses (le martyre pour la cause d'Allah ou la victoire)]...» (Coran 9,52). Et, dans les mots d'un autre mujahid: «Si vous [les infidèles] nous tuez, la vraie foi reste toujours la nôtre / et être tué pour la vérité fera gagner la faveur d'Allah» (Ibn Hicham 1967, 414).

La vertu salvatrice du martyre et l'invincibilité née de la conviction de l'appui de Dieu se complètent dans un autre principe du Coran, à savoir que la force du nombre ne détermine pas le résultat de la guerre. "Ô toi le prophète! Presse les croyants au combat! S'il y a parmi vous vingt hommes patients, ils l'emportent sur deux cents; s'il y en a cent parmi vous, ils en vaincront mille...»(Coran 8,65). Traduit en langue de mujahid, cela devient: "Leur nombre m'est égal aussi longtemps que je vis, je les combattrai par ma fiable épée» (Ibn Hicham 1967, 316).

L'acte du martyr est vu comme un échange spirituel par lequel le musulman offre sa vie pour gagner le paradis. "Ô vous qui croyez! Mettrais-je à votre disposition une marchandise [commerce] pour vous racheter du supplice douloureux? Croyez en Allah et en son prophète, et combattez avec force dans le sentier d'Allah, avec vos biens et vos personnes. Cela est meilleur pour vous, si vous (le) comprenez. » (Coran 61,10-11) " Ce commerce devient davantage exaltant, car Allah lui-même rédime le croyant: "En vérité, Allah a acheté aux croyants leurs personnes et leurs biens pour le Paradis qu'ils auront.» (Coran 9,111) 
En résumé, comme le dit Lawson (1995, 58-59) le martyr se démarque des autres croyants sur plusieurs plans, autant d'aspects renforçant la glorification du sacrifice pour la cause d'Allah:

1) il est exempté de l'interrogation post-mortem;

2) il est exempté du Purgatoire (Barzakh) et prendra comme demeure la meilleure place dans le Paradis tout près d'Allah;

3) cette demeure est qualifiée dans le hadith de la plus belle place et appelée Maison des Martyrs;

4) au Jugement Dernier les blessures des martyrs scintilleront en rouge et sentiront le musc;

5) de tous les habitants du Paradis, ce sont seulement les martyrs qui souhaitent et qui sont autorisés à retourner sur la terre pour se sacrifier de nouveau;

6) un martyr est pardonné de tous ses péchés et n'a pas besoin de l'intercession du Prophète;

7) certains hadiths présentent même les martyrs comme les intercesseurs entre les pécheurs et Allah;

8) lors des funérailles, les martyrs sont enterrés tels quels sans l'ablution rituelle et sans linceul;

9) certains hadiths accordent au martyr le deuxième rang suivant les prophètes.

\section{Le chiisme et le martyre}

\subsection{L'apparition du chiisme}

L'émergence de la division sunnite/chiite date du décès de Mahomet en 632. La question de la succession politique a divisé la communauté musulmane et les compagnons de Mahomet. Ceux qui étaient pour la nomination d'Ali, le gendre et cousin du prophète, furent les premiers chiites ou encore des Alides.

Au fil des siècles, cette dispute initiale concernant le pouvoir politique s'est graduellement transformée en confrontation théologique et doctrinaire. Le chiisme est donc devenu une école islamique très élaborée et sophistiquée, particulièrement grâce à sa synthèse avec la tradition philosophique, mystique et religieuse de la Perse. Ainsi, le chiisme est devenu la religion de l'Empire safavide en Iran en 1501. Dans le reste du monde musulman les empires qui se succèdent - umayyade, abbaside et ottoman — restent sunnites. 
Le chiisme est une branche minoritaire de l'islam, car seulement un musulman sur dix est chiite, mais les musulmans chiites sont majoritaires en Iran et en Irak. Chez les chiites le pouvoir politique et théologique appartient aux Saints qui descendent de l'imam Ali. La branche chiite la plus nombreuse et la mieux connue est celle qui domine en Iran, l'imanisme duodécimain, mais d'autres sectes chiites, beaucoup moins importantes, existent, telles que l'ismaélisme et le zaydisme.

\subsection{Concept de martyre}

Le concept de martyre ne constitue pas une différence fondamentale entre sunnites et chiites. Les deux traditions ont le même fondement, mais le concept de chahada possède des traits particuliers pour le chiisme. Comme le dit Khosrokhavar, "si dans le sunnisme le martyre est associé, voir subordonné, à la guerre sainte, dans le chiisme [...] on rencontre une structure affective centrée sur le martyre, donnant un sens original à la religion d'Allah» (2003, 34-35).

Les chiites ont élargi le sens original du concept de martyre. Cela est vrai dans la mesure où la guerre sainte contre les infidèles n'est plus la seule référence de l'usage du terme chahid. Étant une minorité persécutée et une opposition politique, les chiites n'hésitent pas à utiliser le mot chabid pour des victimes de «la tyrannie sunnite». Ainsi, la tradition martyre chiite s'est construite sur la base de cette opposition au pouvoir sunnite. Cette dimension de la martyrologie chiite la différencie de la terminologie sunnite de martyre. Chez cette dernière le terme renvoie principalement aux martyrs des guerres des conquêtes musulmanes contres les infidèles.

\subsection{Rituel de chahid}

L'idéal de martyre est très présent et glorifié sur le plan discursif et rituel. Dans la tradition chiite, les imams (en commençant par Ali ibn Abi-Talib, premier des Douze Saints, d'où le terme «chiites duodécimains») sont de grands martyrs, tous persécutés ou tués par les califes sunnites. La commémoration annuelle de leur mort fait partie des rites sacrés chiites. La Laylatolghadre (la Nuit du Destin qui se tient pendant le mois de Ramadan) et l'Achoura (le dixième jour du mois de Moharram) sont les deux cérémonies funèbres les plus importantes. La cérémonie de la Nuit du Destin fait référence à l'assassinat de l'imam Ali par une secte musulmane extrémiste, tandis que l'Achoura, la cérémonie la plus populaire et la plus sacrée, repré- 
sente l'époque la plus tragique de l'histoire du chiisme. En effet, Hossein ibn Ali, troisième imam chiite et fils d'Ali, fut massacré avec ses 72 compagnons; leurs familles furent torturées et emprisonnées par le calife umayyade Yazid en 683.

Parlant de la fin tragique de Hossein, Khosrokhavar écrit: "Son martyre devient l'emblème du chiisme duodécimain. Chaque année les croyants célèbrent l'anniversaire de sa mort tout au long du mois de Moharram, avec des processions de flagellants et des mises en scène théâtrales ( $\left.t a^{\prime} z i e h\right)$, marquant les étapes qui aboutissent à sa mort tragique.» (2003, 35) Le martyre de Hossein est un cas paradigmatique, car, pour les chiites, il fonde une tradition pluriséculaire. Pour les sunnites, par contre, c'est un événement triste, signe des conflits entre musulmans, une tache dans l'histoire de l'islam. Pour mieux saisir le sens et la gravité de cet événement, il faut s'imaginer les actes rituels qui accompagnent la commémoration du martyre de Hossein pendant le mois de Moharram. Pour cela, tournons-nous vers Jozani qui écrit:

... dès l'approche de ces jours appelés tâsu'â et 'Achurâ [...] l'anniversaire du martyre de Hoseyn et de ses compagnons [déclarés officiellement comme jours de deuil], les gens portent le noir ou des couleurs sombres et une atmosphère de deuil s'installe partout. On entend, ici et là, aux environs des mosquées, des Hoseyniye (locaux voués aux oraisons funèbres de l'imam Hoseyn), des tekiye (locaux provisoirement aménagés pour les cérémonies [...]), à l'occasion des cérémonies organisées chez les particuliers (rozekhâni), à la radio et à la télévision, des chants appelant ce Martyre. Ceci relève du décor général dans lequel tout un chacun vit ces dix jours. À tout cela, s'ajoutent plusieurs processions de pénitents appelés dasté qui, tout en marchant, chantent le Martyre de Hoseyn et se tapent la poitrine (sinezani), certains se flagellant avec des chaînes (zanjirzani). Les linges blancs maculés de rouge symbolisant le sang, le cercueil de Hoseyn, le berceau de son nourrisson [...] sont les éléments symboliques pour une mise en scène parfaite du drame. Les spectateurs, qui se placent des deux côtés de leur passage, pleurent, chantent, se tapent la poitrine. Cette mise en scène peut donner lieu aux représentations théâtrales populaires nommées ta'ziye (qui peuvent avoir lieu en dehors de cette période de deuil). Pénitents et spectateurs revivent la tragédie de Hoseyn et des siens, et ceci dans ses moindres détails, détails gravés dans la mémoire des Iraniens. (1995, 24-25)

Ces cérémonies de martyre ne sont pas exclusives aux Saints chiites. Certains de ces rites sont repris lors d'autres anniversaires de décès 3 . Par

3. Il est intéressant de noter les liens possibles entre le rituel chiite de commémoration 
exemple, à l'occasion du martyre des soldats iraniens pendant la guerre Iran-Iraq (1980-1988), on reproduit certaines de ces cérémonies pour respecter la mémoire de disparus de la guerre. De plus, l'importance de ces cérémonies est tellement grande dans la culture populaire qu'à l'occasion des décès naturels, on commémore le martyre de Hossein et de ses compagnons pour leur demander la bénédiction divine.

La tragédie d'Achoura est systématiquement utilisée comme idéologie de résistance. Celle-ci vise à la fois à protéger la survie culturelle chiite et à exprimer son opposition au pouvoir sunnite dominant dans le monde musulman.

\subsection{Chiisme, politique et martyre}

Pour les chiites, une interprétation militante du martyre de l'imam Hossein s'oppose à une autre, doloriste.

Dans l'interprétation militante, le martyre de Hossein est idéologisé et sert de base à une révolution religieuse. Le martyre de Hossein devient le symbole de la résistance contre la corruption de la dynastie sunnite omeyyade. Selon cette idéologie, Hossein a donné sa vie à l'islam, a accéléré la chute des Omeyyades et favorisé la montée des Abbasides qui étaient plus proches de la famille du prophète (Khosrokhavar 2004, 36). Trois concepts clé sont au cœur de cette idéologisation: la foi, l'exode et le djihad. C'est pourquoi l'islamisme chiite révolutionnaire prêche une discipline religieuse, le refus de l'ordre injuste, impie et corrompu, et le recours à l'action militaire, élément nécessaire pour changer le statut quo.

Cette idéologisation rend accessible l'action martyre aux musulmans ordinaires, car, par son action révolutionnaire et militante, Hossein est présenté comme un exemple que chacun peut suivre. Hossein n'est plus un simple homme saint, l'objet de deuil et d'admiration (Khosrokhavar 2004, 70), mais devient un leader charismatique et politique qui guide l'action politique. Selon Khosrokhavar, «La tâche d'humanisation et de modernisation de l'imam Hossein a eu, dans les milieux chiites, une grande portée symbolique. La voie était désormais ouverte pour transcrire l'abnégation révolutionnaire dans un idiome religieux, brandir le drapeau de la protestation rehaussé du prestige d'un Hossein contestataire et insoumis » $(2004,70)$.

de la tragédie d'Achoura et la fonction de catharsis propre à la tragédie grecque selon Aristote. 
L'exaltation de l'action martyre atteint le paroxysme idéologique chez Ali Chariati, un intellectuel religieux iranien des années 1960-1970, qui fait une distinction entre le mujahid et le martyre. Alors que le combattant cherche la victoire militaire, le chahid, par son sacrifice physique, est témoin de l'ordre injuste et de la véracité de son message afin d'inspirer les autres pour dépasser la peur (Khosrokhavar 2004, 74-75).

En donnant une lecture plus politique et idéologique au martyre, on alimente l'ardent désir d'une bonne partie de la jeunesse de se porter volontaire dans les opérations militaires. Ainsi, on assiste à la popularisation du sacrifice physique. Le deuil religieux associé au martyre de l'imam Hossein et à ses actions exemplaires et exceptionnelles inspire les actions de combat.

À partir des années 70, l'islamisme chiite a suivi ce cours et donné le ton aux événements les plus marquants des dernières décennies. Que ce soit la Révolution islamique iranienne de 1979, dirigée par l'ayatollah Khomeiny, la guerre Irak-Iran, l'émergence du mouvement Hizbullah chiite au Liban contre l'occupation israélienne, ou, plus récemment, la résistance chiite en Irak, tous ont été fort imprégnés par cette "martyropathie". Encore une fois, cette idéologie, qui réclame un islam révolutionnaire et dihadiste, s'insurge contre un ordre jugé « dépravé, corrompu et injuste». Le martyre de Hossein étant le symbole de mobilisation et de galvanisation des jeunes, l'Achoura prend donc une plus grande portée symbolique et sert d'instrument de mobilisation politique. À titre d'exemple, l'invasion américaine en Irak a permis à certains leaders religieux de la communauté chiite d'exploiter les cérémonies d'Achoura pour mobiliser des milices et organiser des manifestations populaires.

La réaction en chaîne de cette politisation de l'idée de martyre est indéniable. L'islamisme sunnite, tel que celui du Hamas et du Djihad palestiniens, s'est inspiré du culte du martyre chiite pour l'appliquer au conflit arabo-israélien.

En revanche, dans l'interprétation doloriste, la portée politique de l'Achoura reste très limitée. La vision de ses partisans met l'accent sur l'engagement pacifique en faveur de la justice sociale et de la réforme morale et non politique et militante. Pour eux, Achoura est avant tout une pratique religieuse individuelle, rédemptrice et salvatrice. Comme le souligne Khosrokhavar:

Pour les partisans du quiétisme et du dolorisme, la défaite de Hossein signifie que ce monde est une vallée de larmes et que seules les larmes versées pendant le mois de Moharram où on célèbre sa Passion peuvent apporter un 
baume aux maux innombrables que réserve au croyant la vie ici-bas. C'est la version dominante qui, à de rares intervalles près, a constitué la religiosité populaire dans le monde chiite [...] la vision doloriste donnait un sens à la permanence de la répression tout en consolant le croyant par la promesse d'un au-delà de bonheur au paradis. La dissimulation de son appartenance au chiisme (taquié ou ketman) était une attitude fréquente dans les milieux chiites en territoire sunnite. $(2004,75)$

\section{Conclusion}

Modestement, cet article cherchait à présenter aux lecteurs quelques éléments de base afin qu'ils puissent mieux saisir le concept de martyre en islam, son lien avec le djihad et l'acception particulière que le sacrifice physique prend pour le chiisme. La discussion reste cependant préliminaire et une analyse plus approfondie du système de pensée islamique serait indispensable pour mieux décortiquer cette problématique de recherche.

D'autant plus que le contexte international impose une telle recherche. Sur le plan pratique, une relecture de l'islam est nécessaire afin de comprendre le djihad et le martyre dans le contexte des sociétés modernes contemporaines. Étant donnée la couverture médiatique souvent hâtive et trop subjective du monde musulman, associant presque automatiquement islam et terrorisme international, cet exercice épistémologique et herméneutique est impératif. La persistance de certains préjugés négatifs entraîne des effets pernicieux pour la paix sociale. Or, à l'âge de la mondialisation de l'information et de l'interdépendance de nos sociétés, le dialogue et la coexistence ne sauraient que souligner nos ressemblances au lieu d'exacerber nos différences.

\section{Références}

Abu Dawud (1980), Sunan Abi Davud (Traditions reported by Abu Dawud Muhammad Mubi al-Din 'Abd al-Hamid), Beyrouth, al-Maktaba Al'Asryya, vol. 3.

Bukhari, A.M.M. (1974), A Manual of Hadith / préface et traduction par C.E. Bosworth, Londres, Curzon Press.

Coran (1929) / trad. par É. Montet, Paris, Payot.

Dizboni, A.G. (2003), «Le lien entre les concepts de guerre et de martyre au début de l'Islam» Frontières, 15/2, p. 12-16. 
Djaїт, H. (1989), La grande discorde. Religion et politique dans l'Islam des origines, Paris, Gallimard (NRF. Bibliothèque des histoires).

Donner, F.M. (1991), «The Sources of Islamic Conceptions of War », dans J. Kelsay et J. Turner Johnson, dir., Just War and Jihad. Historical and Theoretical Perspectives on War and Peace in Western and Islamic Traditions, New York / Westport, Greenwood Press (Contributions to the Study of Religion 28), p. 31-71.

Ghunaimi, M.T. (1968), The Muslim Conception of International Law and the Western Approach, The Hague, Martin Nijhoff.

Halm, H. (1995) [allemand 1988], Le chiisme / trad. par H. Hougue, Paris, Presses universitaires de France (Islamiques).

Hitтi, P.K. (1968), Makers of Arab History, New York, St. Martin's Press.

Iвn Hicham, A.M. (1967), The Life of Muhammad / trad. par A. Guillaume, Londres, Oxford University Press.

Ibn Khaldoun (1868), Les Prolégomènes / traduit et commenté par M. de Slane, Paris, Imprimerie nationale.

JozAni, N. (1995), "Martyrs au présent: de quelques pratiques populaires à Téhéran ", Frontières, 7/3, p. 24-28.

Khosrokhavar, F. (1995), L'islamisme et la mort: le martyre révolutionnaire en Iran, Paris, L'Harmattan (Comprendre le Moyen-Orient).

(2003), Les nouveaux martyrs d'Allah, Paris, Flammarion (Champs).

Klausner, S.Z. (1987), "Martyrdom», dans M. Eliade et C.J. Adams et al., dir., Encyclopaedia of Religions, New York, McMillan, vol. 9, p. 233234.

LAmmens, H. (1914), Le berceau de l'Islam: l'Arabie occidentale à la veille de l'Hégire, vol. 1: Le climat. Les Bédouins, Rome, Sumptibus Pontificii Instituti Biblici (Scripta Pontificii Instituti biblici).

Lawson, T.B. (1995), "Martyrdom », dans J.L. Esposito, S. Akhavi et al., dir., The Oxford Encyclopedia of the Modern Islamic World, New York / Oxford, Oxford University Press, vol. 3, p. 243-244.

Mılot, J.-R. (1993), L’Islam et les musulmans, Québec, Fides.

Nabhani, Y.I. (1984), Al - Ahadith al-arba' in fi Fadl al-Jihad wa-alMujabidin [Les quarante hadiths sur le statut du djihad], Beyrouth, Dar al-Bashair al-Islamiyya.

Richard, Y. (1991), L’islam chi'ite. Croyances et idéologies, Paris, Fayard. 
SARAKhsi (1989), Commentaire du Grand livre de conduite de l'État (Kitab al-Siar al-Kabir) / trad. par M. Hamidullah, Ankara, Turkiye Diyanet Vakfi, vol. 1.

Shahid, I. (1970), «Pre-Islamic Arabia », dans P.M. Holt, A.K.S. Lambton et B. Lewis, dir., The Cambridge History of Islam, vol. 1A: The Central Islamic Lands From Pre-Islamic Times to the First World War, Cambridge, Cambridge University Press, p. 3-29.

\title{
Résumé
}

Cet article analyse le concept de martyre en islam. En premier lieu, nous présentons brièvement le concept de diihad selon le Coran, les hadiths (les paroles de Mahomet) et les propos des compagnons de ce dernier. En deuxième lieu, nous explorons les caractéristiques du concept de martyre et son lien avec le djihad dans les textes sacrés. En troisième et dernier lieu, nous examinons plus spécifiquement certains aspects rituel, politique et idéologique du martyre dans l'islam et l'islamisme chiites contemporains.

\begin{abstract}
This article examines different aspects of martyrdom in Islam. This analysis follows three steps. Firstly, we briefly present the concept of Jihad and its normative status in Koran, in Hadith and in the statements of the early Muslims. Secondly, we explore the characteristics of the concept of martyrdom and its relationship with Jihad in the Islamic sacred texts. Thirdly and finally, we examine more specifically the ritual, political and ideological characteristics of martyrdom in contemporary Shia Islam.
\end{abstract}

